\title{
Serum Undercarboxylated Osteocalcin Is a Marker of the Risk of Hip Fracture in Elderly Women
}

\author{
P. Szulc, M.-C. Chapuy, P. J. Meunier, and P. D. Delmas \\ Institut National de la Santé et de la Recherche Médicale (INSERM) Unit 234, Hôpital Edouard Herriot, 69437 Lyon Cedex 3, France
}

\begin{abstract}
It has been previously shown that the level of circulating undercarboxylated osteocalcin (ucOC) is elevated in elderly women in comparison with young, healthy, premenopausal ones. To understand the mechanism of the increase in the ucOC in the elderly and to assess its potential consequences on bone fragility, we have measured ucOC in the sera of 195 elderly institutionalized women $70-101$ yr of age. In 45 women ( $23 \%$ ) serum ucOC was above the upper limit of the normal range for young women. The level of ucOC was negatively correlated with 25OHD ( $r=-0.32, P<0.001)$ even after excluding the effect of age, parathyroid hormone (PTH), and creatinine by partial correlation ( $r=-0.24, P<0.002)$. During an 18-mo followup, 15 women sustained a hip fracture and their baseline ucOC level was higher $(P<0.01)$ in women who subsequently sustained hip fracture than in the nonfracture group contrasting with no significant differences for serum calcium, phosphate, alkaline phosphatase, creatinine, PTH, 25OHD, and total and carboxylated $\mathrm{OC}$. The risk of hip fracture was increased in women with elevated ucOC (relative ratio $5.9,99.9 \% \mathrm{Cl} 1.5-$ $22.7, P<0.001$ ). During 1 yr of calcium/vitamin $D_{2}$ treatment, ucOC decreased $(P<0.05)$, especially in those with the initially increased values (from $2.22 \pm 0.35$ to $1.41 \pm 0.29 \mathrm{ng} / \mathrm{ml}, P$ $<0.005)$ contrasting with an increase in the placebo group $(P$ $<0.05$ ). In conclusion, the increase in ucOC in the elderly reflects not only some degree of vitamin $\mathrm{K}$ deficiency but also their poor vitamin D status, suggesting that vitamin D may be important, either directly or indirectly through its effect on bone turnover, for achieving a normal $\boldsymbol{\gamma}$-carboxylation of $\mathrm{OC}$. The ucOC, but not conventional calcium metabolism parameters, predicts the subsequent risk of hip fracture, suggesting that serum ucOC reflects some changes in bone matrix associated with increased fragility. (J. Clin. Invest. 1993.91:17691774.) Key words: $\gamma$-carboxyglutamic acid $\bullet$ osteocalcin • osteoporosis • vitamin D
\end{abstract}

This work was presented in part at the Eleventh International Conference of Calcium Regulating Hormones, 24-29 April 1992, Florence, Italy.

Dr. Szulc's present address is Department of Endocrinology, Medical Center for Postgraduate Education, Bielanski Hospital, 01809 Warsaw, Poland.

Address reprint requests to Dr. P. D. Delmas, Pavillon F, Hopital Edouard Herriot, 69437 Lyon Cedex 3, France.

Received for publication 17 August 1992 and in revised form 14 December 1992

J. Clin. Invest.

(C) The American Society for Clinical Investigation, Inc.

$0021-9738 / 93 / 04 / 1769 / 06 \$ 2.00$

Volume 91, April 1993, 1769-1774

\section{Introduction}

Osteocalcin $(O C)^{1}$ is a bone-specific protein ( 1 ) that circulates in blood. Many studies have shown over the last 10 years that the serum OC level is a sensitive marker of bone turnover that reflects specifically bone formation (2). OC is characterized by the presence of $\gamma$-carboxyglutamic acid residues (GLA). GLA residues are important for the binding of $O C$ to hydroxyapatite and for its presence in bone $(3,4)$. The synthesis of OC depends on both the vitamin D and vitamin $K$. Vitamin D induces directly the $O C$ synthesis by promoting the transcription of its gene (5). Vitamin $\mathrm{K}$ stimulates the posttranslational $\gamma$-carboxylation of glutamic acid residues in the OC propeptide $(1,6)$. Warfarin, a potent vitamin $\mathrm{K}$ antagonist, impairs the $\gamma$-carboxylation of $\mathrm{OC}$, which is reflected by its decreased binding to the hydroxyapatite $(7,8)$.

We have previously shown that the undercarboxylated fraction of circulating $\mathrm{OC}$ ( $\mathrm{ucOC}$ ), i.e., the fraction of $\mathrm{OC}$ which does not bind to hydroxyapatite, is significantly increased in elderly women, suggesting an age-dependent impairment of the $\gamma$-carboxylation of OC (9). The ucOC level was increased slightly after the menopause, but it increased markedly in women after $70 \mathrm{yr}$ of age, accounting for most of the increase in the circulating level of total $O C$ in the elderly. The mechanism of this increase and its potential consequences on bone metabolism are not clear. Low doses of vitamin $\mathrm{K}_{1}(1 \mathrm{mg} /$ day $)$ are able to decrease significantly serum ucOC within 2 wk ( 10 , 11 ), suggesting a role of vitamin $K$ deficiency. One of the major components of circulating vitamin $\mathrm{K}_{2}$, menaquinone-8, is decreased in the elderly (12) and there is growing evidence for vitamin $K_{1}$ and $K_{2}$ deficiency in patients with hip fractures (13-15). Conversely, there is some in vitro evidence that vitamin D might stimulate the $\gamma$-carboxylation of $\mathrm{OC}$ and other GLA-containing proteins of the kidney $(16,17)$. This observation could be relevant to the age-related impairment of the carboxylation of $\mathrm{OC}$, in that vitamin D deficiency is a common feature in the elderly population (18). The present study was designed to address the following questions: $(a)$ Is the increase of circulating ucOC in the elderly women related to vitamin D deficiency? $(b)$ Is the increase in ucOC correlated with an increased risk of osteoporosis, i.e., hip fracture? To answer these two questions, total, carboxylated (carbOC) and ucOC, $25 \mathrm{OHD}$, and other parameters of bone metabolism were measured in a large population of elderly women. The baseline measurements were correlated with the subsequent risk of hip fracture during an 18-mo follow-up. Finally, the effect of a low

1. Abbreviations used in this paper: GLA, $\gamma$-carboxyglutamic acid; $O C$, osteocalcin; similarly carbOC, carboxylated osteocalcin; ucOC, undercarboxylated osteocalcin; also, ucOC\%, undercarboxylated osteocalcin expressed as the fraction of total osteocalcin; ncOC\%, fraction of total osteocalcin that does not bind to hydroxyapatite. 
dose of calcium and vitamin D on the biochemical indices was analyzed.

\section{Methods}

Materials. This study is a part of a large prospective trial on the effect of vitamin $\mathrm{D}$ and calcium treatment on the incidence of hip fracture performed in more than 3,000 elderly women (19). Their physical activity ranged from going outdoors to walking indoors with help. Women with serious medical conditions were excluded as well as women receiving medicines inducing osteoporosis (within the past year), vitamin D and/or calcium (for $>1 \mathrm{yr}$ ), sodium fluoride (for $>3 \mathrm{mo}$ ), and warfarin homologues (at the moment of the recruitment). Local ethical committee approval and an informed personal consent were obtained. The women were randomly assigned to groups treated daily with vitamin $D_{2}, 800 \mathrm{IU}$ daily, and $1.2 \mathrm{~g}$ of the elementary calcium given as calcium phosphate, or to a double placebo. Measurements of baseline levels of OC, PTH, 25OHD, alkaline phosphatase, calcium, phosphorus, and creatinine were performed in 195 patients who were followed prospectively during $18 \mathrm{mo}$. The main goal of the clinical follow-up was to record the occurrence of hip and other nonvertebral fractures (19). In 139 of them ( 71 in the treated group and 68 subjects in the placebo group), the concentration of $\mathrm{OC}$ and its undercarboxylated fraction were also measured after 6 and $12 \mathrm{mo}$. Serum samples were stored frozen at $-30^{\circ} \mathrm{C}$ until the time of the measurement.

The data on the intensity of the sunlight are multiyear average values for the region of Lyon measured in the Meteorogical Laboratory, Lyon-Bron (20). The sun exposure is calculated as the total number of sunny hours per month. The fraction of sun exposure is calculated as the sun exposure divided by the sum of hours between sunrise and sunset for each day during a given month, and expressed in percent. The circannual variation of $25 \mathrm{OHD}$, total and ucOC, and their correlation with the sunlight exposure were evaluated in 260 patients including 195 patients of the study and 65 patients who were lost during the follow-up.

Methods. The OC level was measured with the previously described radioimmunoassay (21) using the rabbit polyclonal antiserum (AS 140 ) which has the same affinity for native $O C$ and thermically decarboxylated OC. The bound OC was precipitated with the mixture of sheep anti-rabbit IgG antiserum and polyethylene glycol (PR CIS BIO Industrie, France) and centrifuged. The precipitate was rinsed with the assay buffer and recentrifuged. The sensitivity of this assay is $0.2 \mathrm{ng} /$ $\mathrm{ml}$. The method of measurement of the ucOC based on different affinity of carbOC and decarboxylated OC for hydroxyapatite, is described in detail elsewhere (21). Briefly, 250-ml samples were incubated with 5 and with $10 \mathrm{mg}$ of hydroxyapatite (calcium phosphate tribasic type IV, Sigma Chemical Co.) in an Eppendorf tube and mixed end-over-end for $1 \mathrm{~h}$ at $+4^{\circ} \mathrm{C}$ and then centrifuged.

These two concentrations of hydroxyapatite were chosen inasmuch as they give the best discrimination between the hydroxyapatite binding of fully carboxylated bovine $\mathrm{OC}$ and thermically decarboxylated bovine OC spiked into an OC-depleted human serum (21). This is a valid estimate of the noncarboxylated fraction of $\mathrm{OC}$, although it should not be regarded as an absolute measurement of the degree of carboxylation of $\mathrm{OC}$, because a significant fraction of fully decarboxylated $O C$ binds to hydroxyapatite (21). The ucOC concentration, calculated as the mean of concentrations of duplicate measurements performed in both supernatants (with 5 and $10 \mathrm{mg}$ of hydroxyapatite), represents the concentration of $\mathrm{OC}$ which is not bound to hydroxyapatite. The ucOC level was also expressed as the percentage of total OC concentration (ncOC\%) and represents the fraction of total $\mathrm{OC}$ that does not bind to hydroxyapatite. The carbOC concentration was calculated as the difference between total $\mathrm{OC}$ and ucOC and is $\mathrm{OC}$ bound to hydroxyapatite. The upper limit of the normal range for $\mathrm{OC}$ and its fractions was calculated as the mean +2 SD in 21 healthy, premenopausal women, aged 21-44 $\mathrm{yr}(9)$. Thus, the upper limits of normal are
$13.9 \mathrm{ng} / \mathrm{ml}$ for total $\mathrm{OC}, 12.5 \mathrm{ng} / \mathrm{ml}$ for carbOC, and $1.65 \mathrm{ng} / \mathrm{ml}$ ( $16.7 \%$ of total) for ucOC. The coefficients of variation $(\mathrm{CV})$ for the unbound fraction of osteocalcin were the following: the intraassay $\mathrm{CV}$ was $6.0 \%$ for $0.38 \mathrm{ng} / \mathrm{ml}$ of ucOC, $8.8 \%$ for $1.08 \mathrm{mg} / \mathrm{ml}, 4.6 \%$ for 1.59 $\mathrm{ng} / \mathrm{ml}$; the interassay $\mathrm{CV}$ was $12.3 \%$ for $0.39 \mathrm{ng} / \mathrm{ml}, 5.3 \%$ for 1.13 $\mathrm{ng} / \mathrm{ml}$, and $9.1 \%$ for $1.64 \mathrm{ng} / \mathrm{ml}$.

The serum concentrations of calcium, inorganic phosphorus, total protein, and creatinine were measured by spectrophotometry. The corrected total plasma calcium level was calculated according the formula described by Parfitt (22). Intact 1-84 PTH concentration was measured using the Magic Lite Intact PTH Immunoassay (CIBA Corning). The $250 \mathrm{OHD}$ level was determined with a radiobinding assay kit (Bühlmann Laboratories AG, Switzerland). The 1 $\alpha, 25$-dihydroxyvitamin $\mathrm{D}\left(1 \alpha, 25(\mathrm{OH})_{2} \mathrm{D}\right)$ level was determined using a radiocompetitive assay kit after an acetonitril extraction (Nichols Institute) in 36 women at baseline and after treatment. The alkaline phosphatase activity was measured using automated analyzer (Boehringer Mannheim GmbH, Mannheim, FRG).

Statistical analysis. Partial correlation coefficients (23) were calculated using the appropriate formulas. For the comparisons between groups and within groups the analysis of variance (ANOVA) and Student's $t$ test (paired and unpaired) were used. The relative hip fracture risk was estimated with the exposure relative ratio ( $R R$ ). The MantelHaensel method (24) of estimation was employed with strata defined by the treatment group (vitamin D plus calcium vs. placebo), presence of diseases (Parkinson's disease, previous stroke, lower limb dysfunction, transient ischemic attacks, paroxysmal arrhythmias, marked dementia, blindness) (25) and treatment with drugs known to increase the risk of hip fracture ( neuroleptics, antidepressors, long half-life anxiolytics) (26).

\section{Results}

Baseline biochemical measurements. Baseline biochemical measurements were compared to normal values established in a group of young healthy women. Serum ucOC was increased in our group of elderly women $(1.22 \pm 1.00$ vs. $0.65 \pm 0.50 \mathrm{ng} /$ $\mathrm{ml}, P<0.01)$. Based on the upper limit of normal of 1.65 $\mathrm{ng} / \mathrm{ml}$ for young healthy women, 45 of the elderly women in this study, i.e., $23 \%$ had an elevated ucOC concentration. Women with a high serum ucOC level had a significantly lower 25OHD level, higher serum PTH, and alkaline phosphatase activity than women with a normal ucOC (Table I). Serum

Table I. Biochemical Characteristics of the Women with Normal (< $1.65 \mathrm{ng} / \mathrm{ml})$ and High (> $1.65 \mathrm{ng} / \mathrm{ml})$ ucOC Concentrations

\begin{tabular}{lccc}
\hline \multicolumn{1}{c}{$\begin{array}{c}\text { Serum } \\
\text { parameter }\end{array}$} & $\begin{array}{c}\text { Normal ucOC } \\
(\mathrm{n}=150)\end{array}$ & $\begin{array}{c}\text { High ucOC } \\
(\mathrm{n}=45)\end{array}$ & Significance \\
\hline Age & $83.0 \pm 0.5$ & $85.9 \pm 1.2$ & $<0.05$ \\
ucOC $(\mathrm{ng} / \mathrm{ml})$ & $0.62 \pm 0.75$ & $2.69 \pm 1.00$ & $<0.0001$ \\
ucOC $(\%)$ & $10.0 \pm 5.7$ & $24.7 \pm 7.1$ & $<0.0001$ \\
Total OC $(\mathrm{ng} / \mathrm{ml})$ & $5.35 \pm 2.73$ & $10.69 \pm 2.79$ & $<0.0001$ \\
Calcium $(\mathrm{mg} / \mathrm{liter})$ & $91.8 \pm 3.7$ & $90.5 \pm 4.3$ & $\mathrm{NS}$ \\
Corrected Ca & $94.1 \pm 3.4$ & $94.1 \pm 4.3$ & $\mathrm{NS}$ \\
Phosphate $(\mathrm{mg} / \mathrm{liter})$ & $34.3 \pm 2.8$ & $35.2 \pm 3.3$ & $\mathrm{NS}$ \\
Creatinine $(\mathrm{mg} / \mathrm{liter})$ & $7.7 \pm 0.2$ & $8.8 \pm 0.6$ & $<0.02$ \\
25OHD $(\mathrm{ng} / \mathrm{ml})$ & $18.3 \pm 13.8$ & $11.6 \pm 8.2$ & 0.003 \\
PTH $(\mathrm{pg} / \mathrm{ml})$ & $47.0 \pm 24.6$ & $62.4 \pm 40.6$ & $<0.005$ \\
Alkaline phosphatase & & & \\
$\quad(I U / l i t e r)$ & $76.3 \pm 31.0$ & $95.0 \pm 41.5$ & $<0.005$ \\
& & & \\
\hline
\end{tabular}


ucOC was correlated positively with $\mathrm{PTH}$, age, and creatinine and negatively with serum $25 \mathrm{OHD}$. Because these parameters are correlated within each other, data were analyzed by partial correlations. A significant negative correlation between ucOC concentration and 250HD level persisted after excluding the effect of PTH, creatinine, and age $(r=-0.24, P<0.002$, Table II). Similarly, ucOC expressed as the percentage of total OC correlated with 25OHD level $(r=-0.21, P<0.01)$. In 36 women, in whom it was measured, $1 \alpha, 25(\mathrm{OH})_{2} \mathrm{D}$ was not significantly correlated with total OC, ucOC, or ucOC\% $(r=0.05$, $r=0.16, r=0.28$, respectively).

Serum 25OHD revealed a circannual rhythm $(F=4.48, P$ $<0.001$ ) with the highest concentrations during the summer (data not shown). An opposite circannual rhythm, with the highest levels during the winter and the lowest ones in summer, was observed for ucOC expressed in $\mathrm{ng} / \mathrm{ml}(F=2.23, P<0.01$, Fig. $1 A)$ and in percent of total $O C(F=4.42, P<0.001$, Fig. 1 $B)$. The circannual variation of total $O C$ was borderline $(F$ $=1.8, P<0.06)$ and that of carbOC was not significant $(F$ $=1.54, P=\mathrm{NS}$ ).

The average monthly 25OHD level correlated both with sun exposure $(r=0.65, P<0.03)$ and with the fraction of sun exposure $(r=0.67, P<0.02)$. Similarly, the average monthly concentration of ucOC correlated both with sun exposure $(r$ $=-0.66, P<0.02)$ and with the fraction of sun exposure $(r$ $=-0.64, P<0.03)$. The average monthly ucOC\% disclosed even stronger correlations both with sun exposure $(r=-0.85$, $P<0.001)$ and the fraction of sun exposure $(r=-0.84, P$ $<0.001)$. Neither total $O C$ nor carbOC correlated with sun exposure or the fraction of sun exposure.

Serum ucOC and the risk of hip fracture. During the 18-mo follow-up of our population, 15 patients had a hip fracture (5.1 fractures per 100 patient-years). As shown in Table III, in patients who subsequently sustained a hip fracture, serum ucOC, but no other baseline clinical and biochemical measurements, was significantly higher than in those without subsequent hip fracture. The hip fracture risk was significantly higher in women with the elevated $(>1.65 \mathrm{ng} / \mathrm{ml})$ ucOC level with a relative risk of 5.9 (99.9\% confidence interval: $1.5-22.7, P$ $<0.001$ ) after adjusting for the group of treatment (vitamin D and calcium vs. placebo), for the presence of associated diseases and for the treatment with drugs increasing the hip fracture risk ( Table IV). Similarly, the adjusted risk of hip fracture was higher in women with the elevated $(>16.7 \%)$ ucOC\% with a relative risk of 3.1 (95\% confidence interval: 1.8-5.9, $P$ $<0.05$ ).

Table II. Correlations of Baseline Serum Levels of ucOC, PTH, 25OHD, Creatinine, and Age in 178 Elderly

Instiutionalized Women

\begin{tabular}{lccc}
\hline Parameter & Corrected for & $r$ & $P$ \\
\hline 25OHD & - & -0.32 & $<0.001$ \\
& Age, PTH creatinine & -0.24 & $<0.002$ \\
Age & - & 0.23 & $<0.002$ \\
& PTH, 25OHD, creatinine & 0.14 & NS \\
PTH & - & 0.29 & $<0.001$ \\
& Age, 25OHD, creatinine & 0.15 & NS \\
Creatinine & - & 0.19 & $<0.01$ \\
& Age, PTH, 25OHD & 0.10 & NS \\
\hline
\end{tabular}
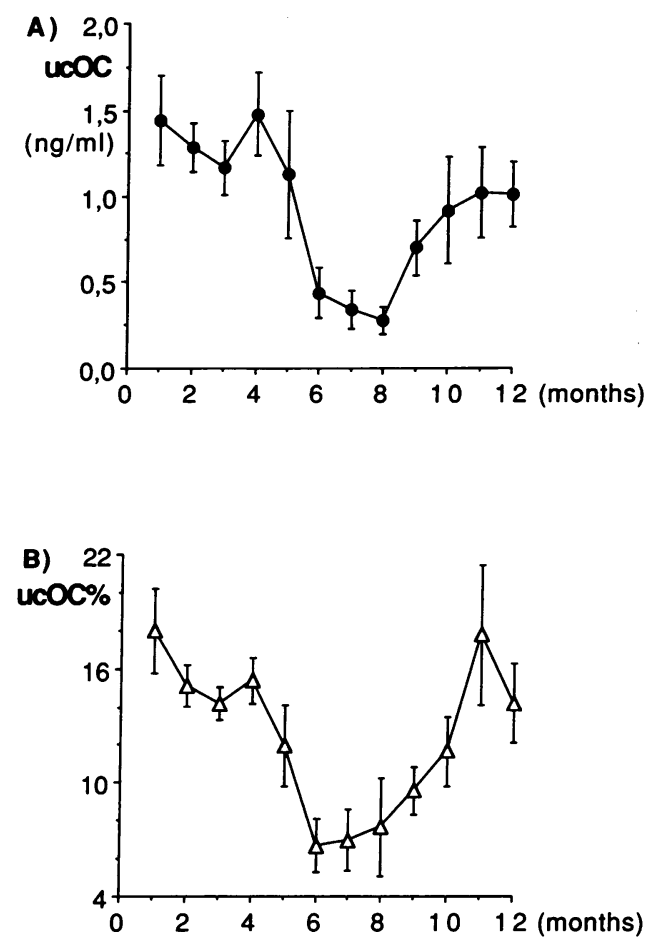

Figure 1. Circannual variation of the concentration of $(A)$ ucOC and $(B)$ ucOC expressed as a fraction of total OC-ucOC\% in 260 elderly women (baseline values). Numbers indicate the months: 1 (January) through 12 (December).

Effect of treatment on ucOC. During the treatment period, total $O C$ was slightly higher in the placebo group than in the group treated with calcium and vitamin $\mathrm{D}$ (Fig. 2). The ucOC concentration increased in the placebo group $(P<0.05)$ and decreased in the treated group $(P<0.05)$ and mean values were significantly different at 6 and $12 \mathrm{mo}$.

Table III. Baseline Biochemical Characteristics of the Subjects Who Subsequently Sustained the Hip Fracture during the Follow-up (Fracture) and Those Who Did Not Fracture (Nonfracture)

\begin{tabular}{lccc}
\hline \multicolumn{1}{c}{$\begin{array}{c}\text { Serum } \\
\text { parameter }\end{array}$} & $\begin{array}{c}\text { Fracture } \\
(n=15)\end{array}$ & $\begin{array}{c}\text { Nonfracture } \\
(n=180)\end{array}$ & $P$ \\
\hline Age & $86.3 \pm 5.6$ & $83.3 \pm 5.1$ & NS \\
Total OC $(n g / m l)$ & $7.8 \pm 4.0$ & $6.4 \pm 3.4$ & NS \\
carbOC $(n g / m l)$ & $6.4 \pm 3.2$ & $5.4 \pm 2.8$ & NS \\
ucOC $(n g / m l)$ & $1.62 \pm 1.16$ & $0.94 \pm 0.93$ & $<0.01$ \\
ucOC\% & $18.6 \pm 11.1$ & $12.6 \pm 8.1$ & $<0.01$ \\
Corrected Ca $(m g / l i t e r)$ & $93.3 \pm 4.8$ & $94.2 \pm 3.5$ & NS \\
Phosphate $(m g / l i t e r)$ & $34.2 \pm 4.3$ & $34.5 \pm 2.8$ & NS \\
Total protein $(g / l i t e r)$ & $66.4 \pm 4.8$ & $69.2 \pm 6.2$ & NS \\
Creatinine $(m g / l i t e r)$ & $9.2 \pm 4.4$ & $7.9 \pm 2.3$ & NS \\
25OHD $(n g / m l)$ & $14.1 \pm 8.3$ & $16.6 \pm 12.4$ & NS \\
PTH $(p g / m l)$ & $61.8 \pm 26.8$ & $49.6 \pm 29.6$ & NS \\
Alkaline phosphatase $(I U / l i t e r)$ & $81.7 \pm 22.2$ & $80.3 \pm 35.2$ & NS \\
& & & \\
\hline
\end{tabular}

Corrected $\mathrm{Ca}$ is the total calcium concentration corrected for the protein level. 
Table IV. Hip Fractures in Subjects with a Normal $(<1.65 \mathrm{ng} /$ $\mathrm{ml}$ ) and Elevated (> $1.65 \mathrm{ng} / \mathrm{ml}) \mathrm{ucOC}$

\begin{tabular}{lrrrr}
\hline \multicolumn{1}{c}{ Group } & \multicolumn{2}{c}{ ucOC } & \\
\cline { 2 - 4 } & $<1.65$ & & $>1.65$ & Total \\
\hline Hip fracture & 7 & & 8 & \\
No fracture & 143 & 37 & 15 \\
Total & 150 & 45 & 180 \\
\hline
\end{tabular}

$\chi^{2}$ with continuity correction $8.2, P<0.005 ; \mathrm{RR} 5.9,99.9 \% \mathrm{Cl} 1.5-$ $22.7, P<0.001$; adjusted for the group (Ca + vitamin $\mathrm{D}$ vs. placebo), cardiovascular and neurological diseases, poor vision, and treatment with psychotropic drugs.

In the calcium/vitamin D-treated group a positive correlation was found between the initial ucOC level and its subsequent decrease $(r=0.62, P<0.001)$. As shown on Fig. 3 , in patients with the initially elevated ucOC level $(>1.65 \mathrm{ng} / \mathrm{ml})$, vitamin $\mathrm{D}$ treatment decreased markedly ucOC concentration expressed in nanograms per milliliter $(P<0.005)$ or in percentage of total OC $(P<0.001)$ whereas ucOC level remained constant in the placebo-treated subgroup. In patients with initially normal ucOC level $(<1.65 \mathrm{ng} / \mathrm{ml})$, ucOC level remained constant in the vitamin $\mathrm{D}$-treated subgroup but rose significantly in the placebo-treated one $(P<0.01)$ although it remained within the normal range of values (Fig. 3). As expected, treatment did not change significantly serum $1 \alpha, 25(\mathrm{OH})_{2} \mathrm{D}$ (from $25 \pm 10$ at baseline to $28 \pm 11 \mathrm{pg} / \mathrm{ml}$ after treatment in the calcium/vitamin D group, from $29 \pm 10$ to $26 \pm 9 \mathrm{pg} / \mathrm{ml}$ in the placebo group).

\section{Discussion}

In this study we have measured the circulating level of ucOC with the hydroxyapatite technique described by Price ( 3 ). This
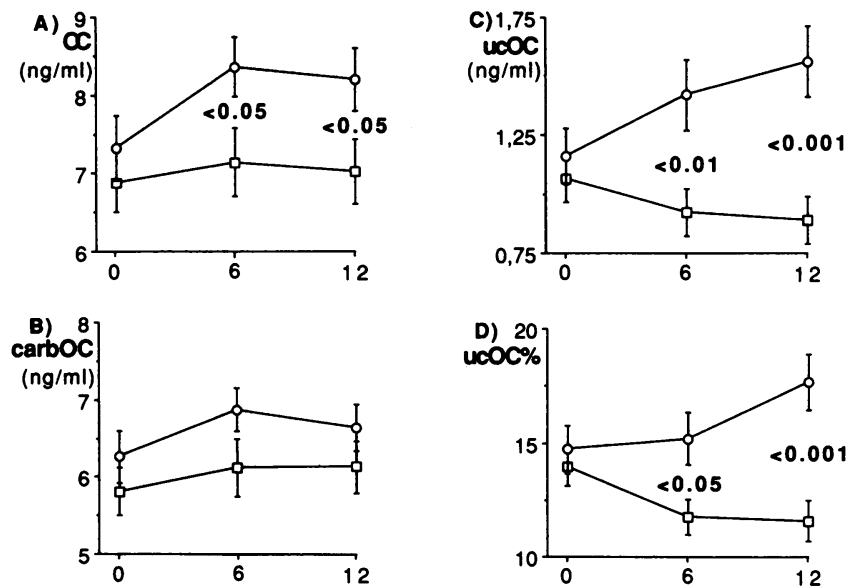

Figure 2. Changes of the serum level of $(A)$ total $O C,(B)$ carbOC and $(C)$ ucOC (expressed in $\mathrm{ng} / \mathrm{ml}$ ) or $(D)$ ucOC\% (percentage of total OC) at 6 and 12 mo of treatment (labeled on the ordinate axis) with calcium and vitamin $\mathrm{D}(\square)$ or placebo $(0) . P$ value indicate differences between groups at a given time.

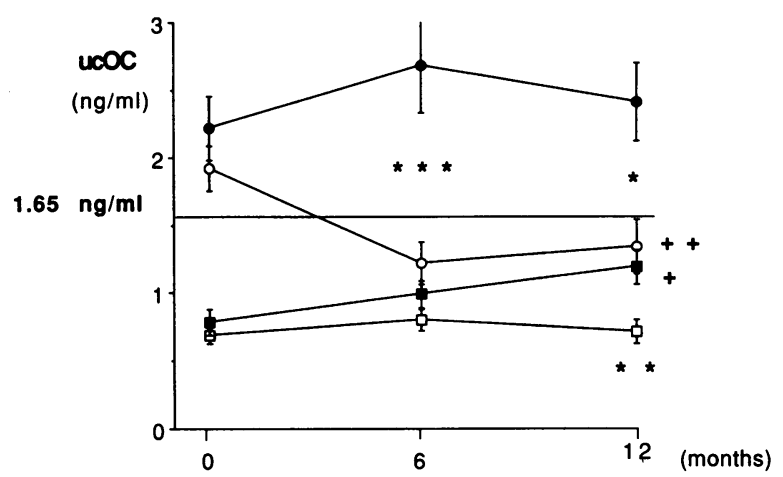

Figure 3. Changes of the serum levels of ucOC in elderly women treated with calcium and vitamin D (open symbols) or placebo (solid symbols) divided into two subgroups according to the initial level of ucOC: (circles) high level ( $>1.65 \mathrm{ng} / \mathrm{ml}$ ); (squares) normal level $(<1.65 \mathrm{ng} / \mathrm{ml})$. Asterisks indicate the significance in the subgroups (treated vs. placebo): ${ }^{*} P<0.005,{ }^{* *} P<0.002,{ }^{* * *} P<0.001$. Plus signs indicate the significance of the change vs. time $0:{ }^{+} P<0.01$, ${ }^{++} P<0.005$.

technique, based on the much higher affinity of carbOC than of thermically decarboxylated $\mathrm{OC}$ for hydroxyapatite, has been validated in rats treated with warfarin, a vitamin $K$ antagonist that inhibits the $\gamma$-carboxylation of OC and other GLA-containing proteins (3). A decreased binding of circulating $\mathrm{OC}$ to hydroxyapatite has also been documented in patients on warfarin therapy $(7-9,21)$. In elderly women with an increased fraction of ucOC, a 2-wk treatment with low doses of vitamin $\mathrm{K}$ induces a significant increase of the hydroxyapatite binding of circulating OC $(10,11)$. These data indicate that the hydroxyapatite-based assay of ucOC is sensitive, although it provides a relative-rather than an absolute-estimate of the degree of carboxylation of circulating OC. Our measurements are based on the average of two incubations of the serum with two concentrations of hydroxyapatite that provide the best discrimination between the binding of carboxylated and fully decarboxylated OC (21). With these assay conditions, a small $(<10 \%)$ of fully carboxylated OC does not bind to hydroxyapatite. More importantly, a significant fraction $(\sim 50 \%)$ of fully decarboxylated $\mathrm{OC}$ binds nonspecifically to hydroxyapatite, indicating that our assay probably underestimates the undercarboxylation of circulating $\mathrm{OC}$ in the elderly. This assay does not permit to distinguish between totally and partially decarboxylated OC moieties. In addition, fragments of $O C$ might circulate, the binding to hydroxyapatite of which has not been characterized. For these reasons, measurement of $\mathrm{OC}$ not bound to hydroxyapatite should be seen as the estimate of the undercarboxylation of $\mathrm{OC}$.

In this study, we have confirmed in a larger population that the circulating levels of ucOC are significantly increased as compared to young healthy women $(10,11)$. As previously mentioned, vitamin $\mathrm{K}$ deficiency is the most likely cause of the subnormal carboxylation of $\mathrm{OC}(7-15)$. The present study suggests that vitamin $D$ deficiency is another important determinant of undercarboxylation of $\mathrm{OC}$ in elderly institutionalized women. First, serum ucOC was negatively correlated with serum 25OHD, even after exclusion of the effect of age, serum PTH, and creatinine. Second, serum ucOC showed a signifcant circannual rhythm that mirrored the one of $250 \mathrm{HD}$, with the average ucOC values three to five times higher in winter 
than in summer. Third, low doses of vitamin D and calcium significantly decreased serum ucOC, particularly in those women with initially elevated values.

Serum $1 \alpha, 25(\mathrm{OH})_{2} \mathrm{D}$ was normal at baseline, did not change significantly with treatment, and did not correlate with either total or ucOC at baseline. Actually, it has been clearly shown that in humans serum $1 \alpha, 25(\mathrm{OH})_{2} \mathrm{D}$ is not a sensitive marker of nutritional vitamin $\mathrm{D}$ deficiency, in contrast to serum 25OHD (27-29). When renal function is not impaired, vitamin D deficiency leads to an increased PTH secretion which in turns stimulates $1 \alpha$-hydroxylation, leading to a compensatory increase of the conversion of $250 \mathrm{HD}$ to $1 \alpha, 25(\mathrm{OH})_{2} \mathrm{D}$. In a recent study performed in osteomalacia, we have also shown that serum OC is a marker of the level of bone turnover, assessed by histomorphometry, and does not correlate with serum $1 \alpha, 25(\mathrm{OH})_{2} \mathrm{D}(29)$. Moreover, in the elderly, low doses of vitamin D increase serum 25OHD but not $1 \alpha, 25(\mathrm{OH})_{2} \mathrm{D}_{3}(19,30-32)$.

The effect of calcium supplementation itself on ucOC cannot be ruled out, inasmuch as our study did not compare a vitamin $\mathrm{D}$ alone treatment group. The combination of calcium and vitamin $\mathrm{D}$ has been shown previously to decrease bone turnover in the elderly, by decreasing the secondary hyperparathyroidism associated with aging $(19,30)$. This increase of bone turnover might affect-through an unidentified mechanism-the degree of carboxylation of OC. Analyzing separately the effect of calcium supplement alone and vitamin D supplementation might be useful to address this issue. Because of the correlation between ucOC and 25OHD at baseline, we believe that vitamin $\mathrm{D}$ supplementation is likely to play a major role in the decrease of ucOC under treatment. As stated above, this effect could be indirect mediated by changes in bone turnover, but it also rises the intriguining possibility that vitamin $\mathrm{D}$ has a direct effect on the $\gamma$-carboxylation of OC. Karl and Friedman (16) described a stimulatory time- and dose-dependent effect of $1 \alpha, 25(\mathrm{OH})_{2} \mathrm{D}_{3}$ on the $\gamma$-carboxylase activity in the microsomes of the renal tubular cells in the rat. In the adult rat, $1 \alpha, 25(\mathrm{OH})_{2} \mathrm{D}_{3}$ stimulates the $\gamma$-carboxylation of the glutamic acid similarly to vitamin K (17). The vitamin D-deficient diet induced a decrease in the GLA content in the femoral bone in the rat (17) but not in the chicken (33). Conversely, in rats on warfarin, administration of $1 \alpha, 25(\mathrm{OH})_{2} \mathrm{D}_{3}$ did not increase the $O C$ content of bone, suggesting that vitamin $D$ cannot counteract the inhibition of $\gamma$-carboxylase activity due to high doses of warfarin (34). In summary, an effect of vitamin $\mathrm{D}$ on the microsomal gammcarboxylase activity is possible but needs to be further documented by in vitro experiments.

The most striking finding in our study was the strong correlation between serum level of ucOC and the subsequent risk of hip fracture during follow-up. The relative risk of hip fracture was six times higher in women with abnormally high value of serum ucOC. In contrast, none of the other biochemical indices had any predictive value for the risk of hip fracture. These data suggest that increased ucOC is associated with increased bone fragility, through a mechanism that remains to be elucidated. In that $\mathrm{OC}$ binds to bone hydroxyapatite through its GLA residues (3), undercarboxylation of OC results in a decrease of the bone matrix content of GLA as shown experimentally in warfarin treated rats $(4,34)$. However, the role of such a decrease on bone metabolism and bone mass is not clear, as the function of osteocalcin is still elusive. $\mathrm{OC}$ is chemotactic for osteoclastic cells and could play a role in the induction of bone resorption and bone turnover (35-37). Chronic warfarin treatment impairs bone development in the rat (38) and has been shown recently to induce bone turnover abnormalities and osteopenia in growing lambs (39) but not in rats $(4,40)$. Bone mineral density has been reported to be slightly but significantly decreased in patients on long-term warfarin therapy (41-43).

In summary, we have found in an elderly population a significant increase of serum ucOC that is a marker of the risk of hip fracture. Serum ucOC was decreased by calcium/vitamin $D$ treatment which has been shown in a much larger population to induce a $30 \%$ decrease of the incidence of hip fracture over $18 \mathrm{mo}$ (19). In the elderly population, especially in those living in institution, poor nutrition, lack of sunlight exposure and skin aging result in both vitamin $D(18,44,45)$ and vitamin $\mathrm{K}$ deficiency (12-15). Our data suggest that ucOC is not only a marker of vitamin $\mathrm{K}$ deficiency but also of vitamin $\mathrm{D}$ deficiency, although the mechanism by which ucOC is linked to bone fragility is yet unclear. It should be stressed that our findings were obtained in a population of institutionalized women, that are vitamin D deficient and therefore not representative of the general population. The predictive value of serum ucOC towards the risk of hip fracture in the general aging population needs to be evaluated.

\section{Acknowledgments}

This work was supported by a grant from the Caisse Nationale d'Assurance Maladie des Travailleurs Salariés-INSERM and the Ministère de la Recherche et de l'Enseignement Supérieur-Aliment 2000 Duphar and Company Laboratories provided the vitamin $\mathrm{D}_{3}$ (Devaron) and Merck-Clevenot Laboratories the tricalcium phosphate (Ostram).

\section{References}

1. Gehron Robey, P. 1989. The biochemistry of bone. Endocrinol. Metab. Clin. N. Am. 18:859-902.

2. Delmas, P. D. 1990. Biochemical markers of bone turnover for the clinical assessment of metabolic bone disease. Endocrinol. Metab. Clin. N. Am. 19:1-18.

3. Price, P. A., M. K. Williamson, and J. W. Lothringer. 1981. Origin of the vitamin K-dependent bone protein found in plasma and its clearance by kidney and bone. J. Biol. Chem. 256:12760-12766.

4. Price, P. A., and M. K. Williamson. 1981. Effects of warfarin on bone Studies on the vitamin $\mathrm{K}$-dependent protein of rat bone. J. Biol. Chem. 256:12754-12759.

5. Lian, J., C. Stewart, E. Puchacz, S. Mackowiak, V. Shalhoub, D. Collart, and G. Zambetti. 1989. Structure of the rat osteocalcin gene and regulation of vitamin D-dependent expression. Proc. Natl. Acad. Sci. USA. 86:1143-1147.

6. Furie, B., and B. C. Furie. 1990. Molecular basis of vitamin K-dependent $\gamma$-carboxylation. Blood. 75:1753-1762.

7. Menon, R. K., D. S. Gill, M. Thomas, P. B. A. Kernoff, and P. Dandonna. 1987. Impaired carboxylation of osteocalcin in warfarin-treated patients. J. Clin. Endocrinol. Metab. 64:59-61.

8. Haarlem, L. J. M. van, M. H. J. Knapen, K. Hamulyak, and C. Vermeer. 1988. Circulating osteocalcin during oral anticoagulant therapy. Thromb. Haemostasis. 60:79-82.

9. Plantalech, L., M. Guillaumont, M. Leclercq, and P. D. Delmas. 1991. Impaired carboxylation of serum osteocalcin in elderly women. J. Bone Miner. Res. 6:1211-1216.

10. Knapen, M. H. J., K. Hamulyak, and C. Vermeer. 1989. The effect of vitamin $\mathrm{K}$ supplementation on circulating osteocalcin (bone Gla protein) and urinary calcium excretion. Ann. Intern. Med. 111:1001-1005.

11. Plantalech, L., M. C. Chapuy, M. Guillaumont, P. Chapuy, M. Leclerq, and P. D. Delmas. 1990. Impaired carboxylation of serum osteocalcin in elderly women: effect of vitamin K1 treatment. In Osteoporosis 1990. C. Christiansen and K. Overgaard. editors. Osteopress, Copenhagen. 345-347.

12. Hodges, S. J., M. J. Pilkington, M. J. Shearer, L. Bitensky, and J. Chayen. 1990. Age-related changes in the circulating levels of congeners of vitamin K2, menaquinone-7 and menaquinone-8. Clin. Sci. 78:63-66. 
13. Hart, J. P., M. J. Shearer, L. Klenerman, A. Catteral, J. Reeve, P. N. Sambrook, R. A. Dodds, L. Bitensky, and J. Chayen. 1985. Electrochemical detection of depressed circulating levels of vitamin $\mathrm{K} 1$ in osteoporosis. J. Clin. Endocrinol. Metab. 60:1268-1269.

14. Hodges, S. J., M. J. Pilkington, T. C. B. Stamp, A. Catterall, M. J. Sheraer, L. Bitensky, and J. Chayen. 1991. Depressed levels of circulating menaquinones in patients with osteoporotic fractures of the spine and femoral neck. Bone. 12:387-389.

15. Hodges, S. J., P. Vergnaud, K. Akesson, K. Obrant, and P. D. Delmas. 1992. Circulating levels of vitamin $\mathrm{K} 1$ and $\mathrm{K} 2$ are decreased in elderly women with hip fracture. Bone Miner. 17(Suppl.):Abstract 16, 73.

16. Karl, P. I., and P. A. Friedman. 1983. Effects of parathyroid hormone and vitamin $\mathrm{D}$ on the renal vitamin $\mathrm{K}$-dependent carboxylating system. J. Biol. Chem. 258:12783-12786.

17. Deyl, Z., and M. Adam. 1983. Evidence for vitamin D dependent $\gamma$-carboxylation in osteocalcin related proteins. Biochem. Biophys. Res. Commun. 113:294-300.

18. Parfitt, A. M., J. C. Gallagher, R. P. Heaney, C. C. Johnston, R. Neer, and G. D. Whedon. 1982. Vitamin D and bone health in the elderly. Am. J. Clin. Nutr. 36:1014-1031.

19. Chapuy, M. C., M. E. Arlot, F. Duboeuf, J. Brun, B. Crouzet, S. Arnaud, P. D. Delmas, and P. J. Meunier. 1992. Vitamin $\mathrm{D}_{3}$ and calcium to prevent hip fracture in elderly women. $N$. Engl. J. Med. 327:1637-1642.

20. Normales Climatologiques 1951-1980. Fascicule 3: Nébulosité, Insolation, Visibilité. 1987. Ministère de l'Équipement du Logement, de l'Aménagement du Territoire et des Transport, Paris, France. No. 4: 196-197.

21. Merle, B., and P. D. Delmas. 1990. Normal carboxylation of circulating osteocalcin (bone Gla-protein) in Paget's disease of bone. Bone Miner. 11:237245.

22. Parfitt, A. M. 1969. Chlorothiazide-induced hypercalcemia in juvenile osteoporosis and hyperparathyroidism. N. Engl. J. Med. 281:55-59.

23. Dagnelie, P. 1973. Théorie et Méthodes Statistiques. Volume 1. Les Presses Agronomiques de Gembloux, Gembloux, Belgium.

24. Rumeau-Rouquette, C., G. Breart, and R. Padieu. 1985. Méthodes en Épidémiologie. Flammarion Médecine-Sciences, Paris. 325-358.

25. Kelsey, J. L., and S. Hoffman. 1987. Risk factors for hip fracture. N. Engl. J. Med. 316:404-406.

26. Ray, W. A., M. R. Griffin, W. Schaffner, D. K. Baugh, and L. J. Melton III. 1987. Psychotropic drug use and the risk of hip fracture. N. Engl. J. Med. 316:363-369.

27. Peacock, M. 1984. Osteomalacia and rickets. In Metabolic Bone and Stone Disease. Nordin. editor. New York. 71-111.

28. Eastwood, J. B., H. E. De Wardener, R. W. Gray, and J. L. Lemann. 1979. Normal plasma $1,25(\mathrm{OH}) 2$ vitamin D concentration in nutritional osteomalacia. Lancet. 1:1377-1378.

29. Demiaux, B., M. E. Arlot, M. C. Chapuy, P. J. Meunier, and P. D. Delmas. 1992. Serum osteocalcin is increased in patients with osteomalacia: correlations with biochemical and histomorphometric findings. J. Clin. Endocrinol. Metab. 74:1146-1151.
30. Chapuy, M. C., P. Chapuy, and P. J. Meunier. 1987. Calcium and vitamin D supplements: effect on calcium metabolism in elderly people. Am. J. Clin. Nutr. 46:324-328.

31. Lips, P., A. Wiersinga, F. C. Van Ginkel, M. J. M. Tongen, J. C. Netelbos, W. H. L. Hackeng, P. D. Delmas, and W. J. F. van der Wijgh. 1988. The effect of vitamin $\mathrm{D}$ supplementation on vitamin $\mathrm{D}$ status and parathyroid function in elderly subjects. J. Clin. Endocrinol. Metab. 67:644-650.

32. Himmelstein, S., T. L. Clemens, A. Rubin, and R. Lindsay. 1990. Vitamin $\mathrm{D}$ supplementation in elderly nursing home residents increases 25OHD but not $1,25(\mathrm{OH})_{2} \mathrm{D}_{3}$. Am. J. Clin. Nutr. 62:701-706.

33. Lian, J. B., M. J. Glimcher, A. H. Roufosse, P. V. Hauschka, P. M. Gallop, L. Cohen-Solal, and B. Reit. 1982. Alterations of the $\gamma$-carboxyglutamic acid and osteocalcin concentrations in vitamin D-deficient chick bone. J. Biol. Chem 257:4999-5003.

34. Price, P. A., and S. A. Sloper. 1983. Concurrent warfarin treatment further reduces bone mineral levels in 1,25-dihydroxyvitamin D3-treated rats. J. Biol. Chem. 258:6004-6007.

35. Lian, J. B., M. Tassinari, and J. Glowacki. 1984. Resorption of implanted bone prepared from normal and warfarin-treated rats. J. Clin. Invest. 73:12231226.

36. Glowacki, J., and J. B. Lian. 1987. Impaired recruitment and differentiation of osteoclast progenitors by osteocalcin-deplete bone implants. Cell Differ. 21:247-254.

37. DeFranco, D. J., J. Glowacki, K. A. Cox, and J. B. Lian. 1991. Normal bone particles are preferentially resorbed in the presence of osteocalcin-deficient bone particles in vivo. Calcif. Tissue Int. 49:43-50.

38. Feteih, R., M. S. Tassinari, and J. B. Lian. 1990. Effect of sodium warfarin on vitamin $\mathrm{K}$-dependent proteins and skeletal development in the rat fetus. $J$. Bone Miner. Res. 5:885-894.

39. Pastoureau, P., P. Vergnaud, P. J. Meunier, and P. D. Delmas. 1990. Osteopenia and bone remodelling abnormalities in warfarin-treated lambs: a possible role of osteocalcin. J. Bone Miner. Res. 5(Suppl.):2:S29. (Abstr. 165).

40. Price, P. A., M. K. Williamson, T. Haba, R. B. Dell, and W. S. S. Jee. 1982 Excessive mineralization with growth plate closure in rats on chronic warfarin treatment. Proc. Natl. Acad. Sci. USA. 79:7734-7738.

41. Fiore, C. E., C. Tamburino, R. Foti, and D. Grimaldi. 1990. Reduced bone mineral content in patients taking an oral anticoagulant. South. Med. J. 83:538-542.

42. Monreal, M., A. Olive, E. Lafoz, and L. del Rio. 1991. Heparins, coumarin, and bone density. Lancet. 338:706

43. Resch, H., P. Pietschmann, E. Krexner, and R. Willvonsender. 1991 Decreased peripheral bone mineral content in patients under anticoagulant therapy with phenprocumon. Eur. Heart J. 12:439-441.

44. Toss, G., S. Almqvist, A. Larsson, and H. Zetterqvist. 1980. Vitamin D deficiency in welfare institutions for the aged. Acta Med. Scand. 208:87-89.

45. Tsai, K-S., H. Heath III, R. Kumar, and B. L. Riggs. 1984. Impaired vitamin D metabolism with aging in women: Possible role in pathogenesis of senile osteoporosis. J. Clin. Invest. 73:1668-1672. 